\title{
Volume reflection and channeling of ultrarelativistic protons in germanium bent single crystals
}

\author{
S. Bellucci ${ }^{1}$ and V. A. Maisheev ${ }^{2}$ \\ ${ }^{1}$ INFN-Laboratori Nazionali di Frascati, Via E. Fermi 40, 00044 Frascati, Italy \\ ${ }^{2}$ Institute for High Energy Physics in National Research Center "Kurchatov Institute," \\ 142281 Protvino, Russia
}

(Received 28 October 2016; published 27 December 2016)

\begin{abstract}
The paper is devoted to the investigation of volume reflection and channeling processes of ultrarelativistic positive charged particles moving in germanium single crystals. We demonstrate that the choice of atomic potential on the basis of the Hartree-Fock method and the correct choice of the Debye temperature allow us to describe the above mentioned processes in a good agreement with the recent experiments. Moreover, the universal form of equations for volume reflection presented in the paper gives a true description of the process at a wide range of particle energies. Standing on this study we make predictions for the mean angle reflection (as a function of the bending radius) of positive and negative particles for germanium (110) and (111) crystallographic planes.
\end{abstract}

DOI: 10.1103/PhysRevAccelBeams.19.121004

\section{INTRODUCTION}

In the last years there were many theoretical and experimental investigations devoted to the study of different processes in bent single crystals. As an example we can point out detailed measurements of such phenomena as volume reflection of ultrarelativistic particles [1-3] and radiation accompanying this process [4] (for electron and positron beams), focusing and mirroring $[5,6]$ effects and others. As expected these investigations will be useful in using high energy particles interactions with bent single crystals for different applications in the accelerator practice. In particular, a special interest lies in the possibility to use such crystals for collimation and extraction of proton and nuclear beams for LHC. It should be noted that most of the investigations with bent crystals were performed with silicon samples. However, in the article [7] the experimental results of the deflection of high energy protons in a germanium single crystal were presented. In this experiment a high enough deflection efficiency was obtained.

Recently new experiments with germanium bent single crystals were performed. Short germanium crystals were used and such phenomena as volume reflection and channeling of $400 \mathrm{GeV}$ protons were investigated [8,9]. However, the analysis of the results of measurements for volume reflection is practically absent and, for channeling, authors give calculations on the basis of Moliere model for atomic potentials.

Published by the American Physical Society under the terms of the Creative Commons Attribution 4.0 International license. Further distribution of this work must maintain attribution to the author(s) and the published article's title, journal citation, and DOI.

\section{CHOICE OF POTENTIAL FOR GERMANIUM CRYSTAL}

Volume reflection represents a coherent scattering of relativistic charged particles in the planar electric field of the bent single crystal. This effect was found in Monte Carlo simulations in Ref. [10]. The analytical consideration of this process was presented in the paper [11]. This theory gives a very good agreement with experimental data for (110) and (111) interplanar fields in silicon single crystals (see Ref. $[12,13])$. However, this good agreement takes place when in calculations for atomic electric fields the data derived from x-ray measurements are used. Calculations based on the popular Moliere model of the potential give worse results.

We began our simulations of coherent processes in germanium single crystals from finding the mean angle of volume reflection. The first calculations were performed for Moliere model of atomic potentials. However, the computed mean angles of volume reflection valuably exceed the measured ones. After this we applied the atomic potential based on the Hartree-Fock method [14,15] to solve our problem. In $[14,15]$ the results of calculating atomic form factors (in the Hartree-Fock method) are presented in the form

$$
F(q)=c_{0}+\sum_{i=1}^{4} a_{i} \exp \left[-b_{i}\left(q^{2} /(4 \pi)\right]\right.
$$

where $a_{1}=16.0816, a_{2}=6.3747, a_{3}=3.7068, a_{4}=3.683$, $b_{1}=2.8509 \AA^{2}, b_{2}=0.2516 \AA^{2}, b_{3}=11.4468 \AA^{2}, b_{4}=$ $54.7625 \AA^{2}, c_{0}=2.1313$ are constants and $\mathbf{q}$ is called the scattering vector. The relations for calculations of planar electric characteristics can be found in Ref. [16]. However, 
for calculations of the interplanar potentials we should know the mean squared amplitude of thermal vibrations of germanium atoms. We can find this value from such an important characteristic of single crystal as its Debye temperature. If we know the Debye temperature $T_{D}$ we can find the mean squared amplitude of atomic thermal vibrations [17]

$$
\left\langle u^{2}\right\rangle=\frac{3 \hbar^{2}}{4 M_{a} k_{B} T_{D}}\left[1+4\left(T / T_{D}\right)^{2} \int_{0}^{T_{D} / T} \frac{y d y}{e^{y}-1}\right]
$$

where $M_{a}$ is the atom mass, $\hbar, k_{B}$ are the Planck and Boltzmann constants, and $T$ is the crystal temperature. The Debye temperature for any substance may be determined experimentally with the help of different methods. As pointed out in the paper [18] there is a difference in Debye temperatures obtained from a specific-heat measurement and from measurements of x-ray Bragg reflections. Besides, the authors of the paper [18] state that the Debye temperature obtained from $\mathrm{x}$-ray measurements is coupled with the thermal atomic vibrations and hence this value should be used in calculations of the mean square of thermal vibrations in the crystal. For silicon and germanium the Debye temperatures are approximately $640^{\circ} \mathrm{K}$ and $360^{\circ} \mathrm{K}$ (and correspond to 0.0645 and $0.068 \AA$ of rms of thermal vibrations) from a specific-heat measurement and $543^{\circ} \mathrm{K}$ and $290^{\circ} \mathrm{K}$ from x-ray measurements (and correspond to 0.0747 and $0.0835 \AA$ of rms of the thermal vibrations).

\section{EQUATION FOR VOLUME REFLECTION IN GENERALIZED VARIABLES}

In the paper [11] it was shown that the main equations describing the volume reflection may be presented in generalized parameters $\Xi=\langle\alpha\rangle / \theta_{c}$ and $\kappa=U_{0} R /\left(E_{0} \beta^{2} d\right)=$ $R / R_{0}$ where $\langle\alpha\rangle$ is the mean volume reflection angle, $\theta_{c}=$ $\sqrt{2 U_{0} /\left(E_{0} \beta^{2}\right)}$ is the critical channeling angle (for unbent single crystal) $R / R_{0}$ is the ratio of bending radius to characteristic radius $R_{0}=\beta^{2} E_{0} d / U_{0}$. Here $E_{0}$ is the particle energy, $\beta$ is the ratio of a particle velocity to velocity of light, $U_{0}$ is the value of potential barrier for unbent crystal, and $d$ is the interplanar distance.

For the first time here we present the explicit relation for the mean volume reflection angle in the generalized variables

$$
\begin{aligned}
\frac{\langle\alpha(\kappa)\rangle}{\theta_{c}}= & \frac{1}{\kappa} \int_{\nu}^{\nu+1} d \nu\left\{\int _ { y _ { 0 } } ^ { y _ { c } } \left[\frac{1}{\sqrt{\nu / \kappa-U(y) / U_{0}-y / \kappa}}\right.\right. \\
& \left.\left.-\frac{1}{\sqrt{\nu / \kappa-U\left(y_{c}\right) / U_{0}-y / \kappa}}\right] d y\right\}
\end{aligned}
$$

where $\nu=E / \delta E$ and $E / U_{0}=\nu / \kappa=E_{0} \beta^{2} \theta_{0}^{2} /\left(2 U_{0}\right)+$ $U\left(y_{0}\right) / U_{0}+y_{0} / \kappa, \quad \delta E=E_{0} \beta^{2} d / R, \quad y=x / d$, and the critical point $y_{c}$ is found from the relation $\frac{\nu}{\kappa}-\frac{U\left(y_{c}\right)}{U_{0}}-\frac{y_{c}}{\kappa}=0$. The main contribution in the inner integral brings the area near the $y_{c}$-point (see [11]). This fact should be taken into account in calculations. For a thick single crystal $\left(l_{0} \gg\langle\alpha\rangle R\right.$, where $l_{0}$ is a thickness of a crystal) the area of large coordinates $y=x / d$ does not give contribution in the inner integral (when $\left|x_{0}-x_{c}\right| \gg\langle\alpha\rangle R^{2}$ ).

Let us call the function $U(y) / U_{0}$ as the normalized potential. From Eq. (3) it follows that the value $\frac{\langle\alpha(\kappa)\rangle}{\theta_{c}}$ depends mainly on the form of the normalized potential (for thick enough single crystals). One can expect that if the normalized potentials for different planes or different single crystals differ slightly in between, then the functions $\Xi\left(R / R_{0}\right)$ for corresponding cases will also differ insignificantly in between. We recall that $U(x)$ is a periodic function with the period equal to $d$.

\section{CALCULATIONS OF VOLUME REFLECTION AND PLANAR CHANNELING FOR GERMANIUM CRYSTAL}

Figure 1 illustrates the results of calculations [with the help of Eq. (3)] of $\Xi\left(R / R_{0}\right)$-functions for normalized potentials which were shown in insert (a). We select for calculations the Hartree-Fock potential (at $T_{D}=290^{\circ} \mathrm{K}$

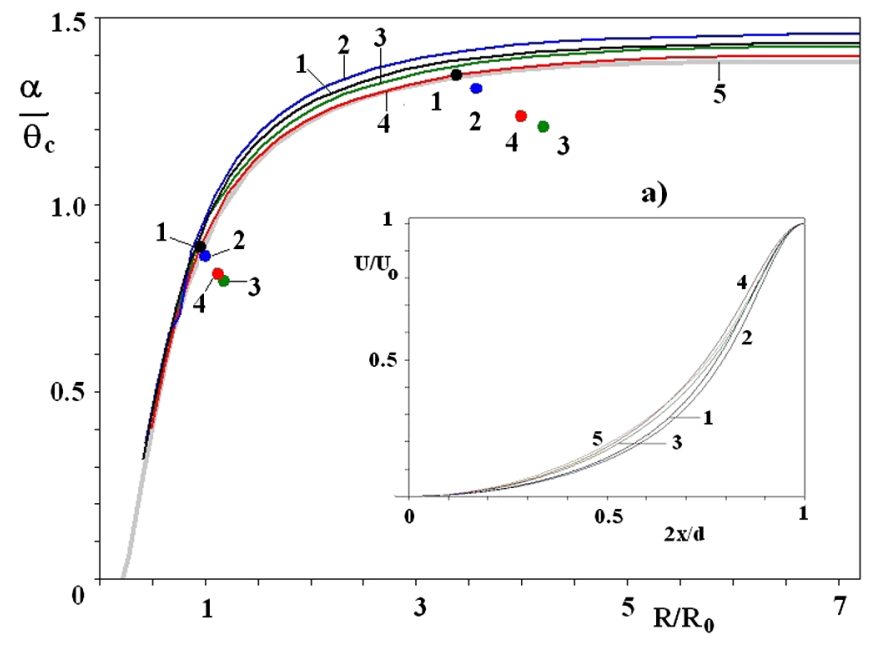

FIG. 1. The calculated universal $\Xi\left(R / R_{0}\right)=\langle\alpha\rangle / \theta_{c}$-functions for positively charged particles and for normalized potentials for (110) plane of germanium which were shown in insert (a). The calculations were done for the Hartree-Fock potential at $T_{D}=$ $290^{\circ} \mathrm{K}$ and at $T_{D}=360^{\circ} \mathrm{K}$ (the curves 1 and 2 , correspondingly) and for the Moliere potential at $T_{D}=360^{\circ} \mathrm{K}$ and at $T_{D}=$ $285^{\circ} \mathrm{K}$ (the curves 3 and 4, correspondingly). All the curves are functions of generalized variable $\kappa=R / R_{0}$, The small circles are the experimental data [9] presented for corresponding values of generalized variables $\Xi$ and $\kappa$. The table I contains the necessary (for such presentation) quantities $\theta_{c}$ and $U_{0}$. The experimental errors are less than \pm 0.02 (for $\Xi$-variable). The curve number 5 [in insert (a)] corresponds to (110) plane of silicon and it is presented for comparison. 
TABLE I. Characteristics of germanium crystal and volume reflection and channeling processes for (110) plane. In the 1st column the model of potential (M-Moliere, HF-Hartree-Fock) and Debye temperature are presented. Other designations are: $U_{0}$ and $\theta_{c}$ are the potential barrier and critical angle of channeling (for $400 \mathrm{GeV}$ ) in straight crystal; the value $\left\langle u^{2}\right\rangle$ is defined by Eq. (2); $\left\langle\alpha_{1}\right\rangle$ and $\left\langle\alpha_{2}\right\rangle$ are the mean angle of volume reflection (at $R=2.3 \mathrm{~m}$ and $8.2 \mathrm{~m}$, correspondingly); $\Xi_{1}$ and $\Xi_{1}^{e}$ are the values of generalized function in Eq. (3) at $R=2.3 \mathrm{~m}$ from calculation and experimental results, correspondingly, and $\Xi_{2}$ and $\Xi_{2}^{e}$ are the same as previous but at $R=8.3 \mathrm{~m}$; $\kappa_{1}$ and $\kappa_{2}$ are the values of generalized variable at $R=2.3 \mathrm{~m}$ and $8.2 \mathrm{~m}$, correspondingly; $\theta_{1}^{a}$ and $\theta_{2}^{a}$ the angle acceptance of bending plane at $R=2.3 \mathrm{~m}$ and $8.2 \mathrm{~m}$, correspondingly. Experimental data from [9].

\begin{tabular}{lccccccccccccc}
\hline \hline Potential & $U_{0}, \mathrm{eV}$ & $\sqrt{\left\langle u^{2}\right\rangle}, \AA$ & $\theta_{c}, \mu \mathrm{rad}$ & $\left\langle\alpha_{1}\right\rangle, \mu \mathrm{rad}$ & $\kappa_{1}$ & $\Xi_{1}$ & $\Xi_{1}^{e}$ & $\left\langle\alpha_{2}\right\rangle, \mu \mathrm{rad}$ & $\kappa_{2}$ & $\Xi_{2}$ & $\Xi_{2}^{e}$ & $\theta_{1}^{a}$ & $\theta_{2}^{a}$ \\
\hline$M, T_{D}=360^{\circ}$ & 41.02 & 0.068 & 14.32 & 14.74 & 1.179 & 1.029 & 0.796 & 20.02 & 4.202 & 1.398 & 1.208 & 11.5 & 13.5 \\
$M, T_{D}=285^{\circ}$ & 39.02 & 0.085 & 13.97 & 13.75 & 1.121 & 0.984 & 0.816 & 19.11 & 3.997 & 1.368 & 1.238 & 11.1 & 13.1 \\
$H F, T_{D}=360^{\circ}$ & 34.81 & 0.068 & 13.19 & 12.74 & 1.000 & 0.966 & 0.864 & 18.64 & 3.567 & 1.413 & 1.312 & 10.4 & 12.4 \\
$H F, T_{D}=290^{\circ}$ & 33.04 & 0.0835 & 12.85 & 11.88 & 0.949 & 0.925 & 0.887 & 17.80 & 3.426 & 1.385 & 1.346 & 9.9 & 11.9 \\
exp. data & $\cdots$ & $\cdots$ & $\cdots$ & $11.4 \pm 0.2$ & $\cdots$ & $\cdots$ & $\cdots$ & $17.3 \pm 0.3$ & $\cdots$ & $\cdots$ & $\cdots$ & $8.8 \pm 0.8$ & $10.9 \pm 0.8$ \\
\hline \hline
\end{tabular}

and at $\left.T_{D}=360^{\circ}\right) \mathrm{K}$ and Moliere potential (at $T_{D}=360^{\circ} \mathrm{K}$ and at $T_{D}=285^{\circ} \mathrm{K}$ ) and numbers of curves (from 1 to 4 ) in the figure take place in the corresponding order. The calculations were done for $l_{0} \approx 2 \mathrm{~mm}[8,9]$. We select $T_{D}=285^{\circ} \mathrm{K}$ (for Moliere potential) because then $\left\langle u^{2}\right\rangle^{1 / 2}=0,085 \AA$ as in the paper [9].

As we predicted, the calculated $\Xi\left(R / R_{0}\right)$-functions are close in between. Then we take the experimental data [9] which was obtained for proton energy $E_{0}=400 \mathrm{GeV}$ and found quantities $\theta_{c}$ and $R / R_{0}$ for every normalized potential. After that we also find values $R / R_{0}$ for the $\mathrm{x}$-axis and $\Xi\left(R / R_{0}\right)$ for the $\mathrm{y}$-axis. Thus, for every potential we calculate the corresponding experimental point. From the figure we see that for Moliere potentials the obtained two points are far enough from corresponding calculated curves (curves number 3 and 4). The curve 1 is close enough to corresponding points with number 1 . We think the fact that both experimental points marked as 1 lie near the curve 1 is important and means that the relative location of these points has a natural (regular) character. These results we also present in Table I for two values of the radius.

A similar procedure was made also for (111) planar potentials of germanium single crystal. As a result we got that the experimental data [which was measured only for one bending radius (equal to 15 meters)] are in a good agreement with the Hartree-Fock potential and $T_{D}=$ $290^{\circ} \mathrm{K}[\langle\alpha\rangle=16.33 \mu \mathrm{rad}$ (calculations) and $\langle\alpha\rangle=15.9 \pm$ $0.3 \mu \mathrm{rad}$ (experiment)].

The results of calculating the mean angle reflection presented here for different atomic models and Debye temperatures and their comparison with experiment show that a good agreement is observed for the atomic model on the basis of Hartree-Fock model and Debye temperature equal to $290^{\circ} \mathrm{K}$. In principle this is an expected result. Really, in the calculations the Hartree-Fock model was applied for every sort of atoms, taking into account some specific atomic properties. The recommendation for the value of $T_{D}$ for germanium follows from paper [18] and stands upon X-ray measurements and theoretical consideration.
We see that the process of volume reflection in germanium may be described well enough with the help of the Hartree Fock potential at $T_{D}=290^{\circ} \mathrm{K}$. Besides, our calculation of some channeling characteristics in this potential should also be compared with experiments. The deflection efficiency has been measured experimentally (see [9]) as a function of the incoming angle relative to the (110) crystallographic plane of germanium. The particles with an incoming angle in $\mathrm{a} \pm 2 \mu \mathrm{rad}$ interval around a given value are selected; the efficiency of deflection for those particles was then computed as the ratio of the number of deflected particles to their total number.

We made the calculations of deflection efficiency for the germanium crystal for the (110) plane and different potential models for 2.3 and 8.2 meter bending radii. This calculation was performed by Monte Carlo method and on the basis of a one-trajectory approximation of a diffusion process which was developed in Ref. [13]. We got the following results for zero incoming angle: for Hartree-Fock potentials the deflection efficiencies are 77.5 and $54\left(\right.$ at $T_{D}=290^{\circ} \mathrm{K}$ ) and 81 and 59.5 (at $T_{D}=360^{\circ} \mathrm{K}$ ) percents, and for Moliere potential the deflection efficiencies are 78.5 and 61 (at $T_{D}=285^{\circ} \mathrm{K}$ ) and 81.5 and 65 (at $T_{D}=360^{\circ}$ ) percents. Here the first value in every pair of quantities corresponds to a bending radius equal to 8.2 meter and the second one corresponds to 2.3 meter for the bending radius. The corresponding measured values are $(74.1 \pm 1.5) \%$ for $R=8.2 \mathrm{~m}$ and $(56 . \pm 1.5) \%$ for $R=2.3 \mathrm{~m}$.

Besides, in Table I the angle acceptance of bending (110) germanium plane is compared with the experimentally defined one. In this case we see a satisfactory agreement.

As a result of our consideration we have demonstrated that the choice of Hartree-Fock potential and the corresponding Debye temperature allows one correctly to describe channeling and volume reflection processes in germanium single crystals with agreement with experimental data, We present our calculations in a universal form which may be used at various initial conditions (see Fig. 2). From here it follows that the mean angle of volume reflection is $\approx 1.3$ larger for germanium in comparison with silicon at 


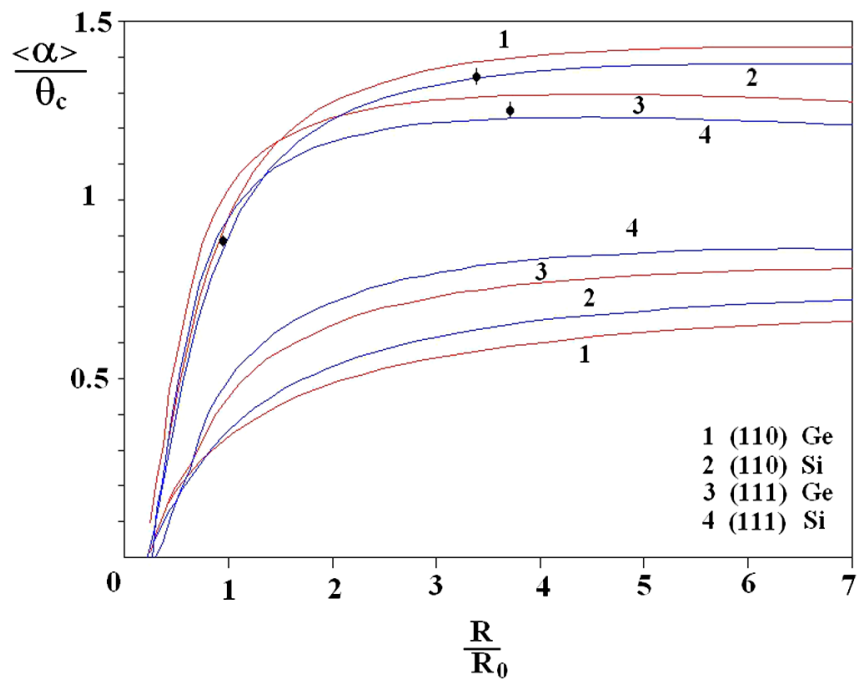

FIG. 2. The calculated universal functions $\Xi\left(R / R_{0}\right)=\langle\alpha\rangle / \theta_{c}$ for germanium and silicon single crystals. The Hartree-Fock potentials were used at $T_{D}=290^{\circ} \mathrm{K}$ and $640^{\circ} \mathrm{K}$ for germanium and silicon, respectively. The upper curves are results for positively charged particles and lower ones are results for negatively charged particles. Three points demonstrated experimental data for germanium crystal [8,9] for $R=2.3,8.2$ and 15 meters and $l_{0}=2 \mathrm{~mm}$ (from left to right, correspondingly). The first and second experimental points correspond to the curve number 1, and the third points correspond to the curve number 3. $R_{0}[\mathrm{~m}]=K_{R} E_{0}[\mathrm{GeV}]$, where $K_{R}=0.0061,0.01$ for (110) and (111) planes in germanium and $K_{R}=0.009,0.014$ for (110) and (111) planes in silicon, respectively.

the same beam energy. Despite the small mean angle of volume reflection we expect a valuable gain when using this phenomenon in multistrip crystal systems $[19,20]$.

\section{CONCLUSION}

In conclusion, more precise atomic potentials than the Moliere model improve the calculations of coherent phenomena in single crystals. For the example considered, the Hartree-Fock potential with a Debye temperature of $290 \mathrm{~K}$ best describes the experimental results.

[1] Yu. M. Ivanov et al., Volume Reflection of a Proton Beam in a Bent Crystal, Phys. Rev. Lett. 97, 144801 (2006).

[2] Yu. M. Ivanov et al., Volume reflection of $1-\mathrm{GeV}$ protons by a bent silicon crystal, JETP Lett. 84, 372 (2006).

[3] W. Scandale et al., High-Efficiency Volume Reflection of an Ultrarelativistic Proton Beam with a Bent Silicon Crystal, Phys. Rev. Lett. 98, 154801 (2007).
[4] W. Scandale et al., Experimental study of the radiation emitted by $180 \mathrm{GeV} / \mathrm{c}$ electrons and positrons volumereflected in a bent crystal, Phys. Rev. A 79, 012903 (2009).

[5] W. Scandale et al., Observation of focusing of $400 \mathrm{GeV} / \mathrm{c}$ proton beam with the help of bent crystals, Phys. Lett. B 733, 366 (2014); Corrigendum, Phys. Lett. B 734, 408 (2014).

[6] W. Scandale et al., Mirroring of $400 \mathrm{GeV} / \mathrm{c}$ protons by an ultra-thin straight crystal, Phys. Lett. B 734, 1 (2014).

[7] C. Biino et al., Deflection of $200 \mathrm{GeV} / \mathrm{c}$ and $450 \mathrm{GeV} / \mathrm{c}$ positively charged particles in a bent germanium crystal, Phys. Lett. B 403, 163 (1997).

[8] D. De Salvador et al., Steering of an ultrarelativistic proton beam by a bent germanium crystal, Appl. Phys. Lett. 98, 234102 (2011).

[9] D. De Salvador et al., Highly bent (110) Ge crystals for efficient steering of ultrarelativistic beams, J. Appl. Phys. 114, 154902 (2013).

[10] A. M. Taratin and S. A. Vorobiev, "Volume reflection" of high-energy charged particles in quasi-channeling states in bent crystals, Phys. Lett. A 119, 425 (1987).

[11] V. A. Maisheev, Volume reflection of ultrarelativistic particles in single crystals, Phys. Rev. Accel. Beams 10, 084701 (2007).

[12] W. Scandale et al., Volume Reflection Dependence of 400 GeV/c Protons on the Bent Crystal Curvature, Phys. Rev. Lett. 101, 234801 (2008).

[13] S. Bellucci, Y. A. Chesnokov, V. A. Maisheev, and I. A. Yazynin, Volume reflection and volume capture of ultrarelativistic particles in bent single crystals, Phys. Rev. Accel. Beams 18, 114701 (2015).

[14] http://lamp.tu-graz.ac.at/ hadley/ss1/crystaldiffraction/ atomicformfactors/formfactors.php.

[15] P. J. Brown, A. G. Fox, E. N. Maslen, M. A. O'Keefe and B. T. M. Willis, Intensity of diffracted intensities, International Tables for Crystallography (2006), Vol. C, ch. 6.1 , p. 554 .

[16] E. Bagli, V. Guidi, and V. A. Maisheev, Calculation of the potential for interaction of particles with complex atomic structures, Phys. Rev. E 81, 026708 (2010).

[17] M. Ter-Mikaelyan, High Energy Electromagnetic Processes in Condensed Media (Wiley-Interscience, New York, 1972).

[18] B. W. Batterman and D. R. Chipman, Vibrational Amplitudes in Germanium and Silicon, Phys. Rev. 127, 690 (1962).

[19] W. Scandale et al., Deflection of high energy protons by multiple volume reflections in a modified multi-strip silicon deflector, Nucl. Instrum. Methods Phys. Res., Sect. B 338, 108 (2014).

[20] S. Bellucci, Y. A. Chesnokov, P. N. Chirkov, M. Cosic, G. Giannini, V. A. Maisheev, S. Petrovic, and I. A. Yazynin, Deflection of a $100-\mathrm{MeV}$ positron beam by repeated reflections in thin crystals, JETP Lett. 98, 649 (2014). 